\section{Cyclophosphamide versus azathioprine for proliferative lupus nephritis therapy}

There is currently no consensus as to whether cyclophosphamide is better than azathioprine, in combination with corticosteroids, for the treatment of proliferative lupus nephritis-although azathioprine has less gonadal toxicity.

In this open-label trial, 87 patients with proliferative lupus nephritis were randomly allocated to 2 years of treatment with either intravenous pulsed cyclophosphamide ( $750 \mathrm{mg} / \mathrm{m}^{2}, 13$ pulses) plus oral prednisone, or to oral azathioprine ( $2 \mathrm{mg} / \mathrm{kg}$ daily) plus intravenous pulsed methylprednisolone $(3 \times 3$ pulses of $1 \mathrm{~g})$ and oral prednisone. The 2-year incidence of partial or complete renal remission was similar in both groups. After a median follow-up of 5.7 years, however, doubling of initial serum creatinine level occurred more frequently in azathioprine-treated than in cyclophosphamide-treated patients (although the difference was not statistically significant), and relapses occurred more often in azathioprine-treated patients, which indicated that cyclophosphamide had greater efficacy than azathioprine. There were also more infections (mostly herpes zoster) in azathioprinetreated than in cyclophosphamide-treated patients; the authors attribute this finding to the higher corticosteroid doses given with azathioprine. Indicators of ovarian function were similar in both groups, but the four women who developed premature ovarian failure had all received cyclophosphamide. After a median follow-up of 6.3 years, $88.4 \%$ of the azathioprine-treated patients had not required cyclophosphamide treatment, which consequently improved their prognosis, in terms of fertility.

Longer follow-up is needed before final conclusions can be drawn in relation to the effects of these two regimes on renal function.

Original article Grootscholten C et al. (2006) Azathioprine/ methylprednisolone versus cyclophosphamide in proliferative lupus nephritis. A randomized controlled trial. Kidney Int 70: 732-742

\section{Daily COX2-inhibitor use might increase fracture risk in men}

Cyclo-oxygenase 2 (COX2) regulates the production of prostaglandin $E_{2}$. Inhibition of COX2 reduces prostaglandin $\mathrm{E}_{2}$ levels, which inhibits bone formation in response to increased mechanical loads, and improves the proinflammatory state associated with postmenopausal bone loss. Richards and colleagues, therefore, investigated the effect of COX2-inhibitor use on bone mineral density (BMD). They found that men who used COX2 inhibitors daily had reduced BMD. Conversely, postmenopausal women who used COX2 inhibitors daily but did not use estrogen supplementation had increased BMD. Daily COX2-inhibitor use had no net effect on BMD in postmenopausal women who used estrogen replacement.

Richards and colleagues evaluated data from 2,004 men (aged $>25$ years) and 2,776 postmenopausal women (aged $\geq 65$ years) from the Canadian Multicentre Osteoporosis Study. Participants' BMD was assessed at enrollment and after 5 years. Daily COX2-inhibitor use was reported by 394 individuals, and daily aspirin use by 1,109 individuals. Among daily users of COX2 inhibitors, BMD of the hip and spine declined by $3.1 \%(95 \% \mathrm{Cl} 2.4-5.3 \%)$ in men, and increased by $3 \%(95 \% \mathrm{Cl} 0.9-5.7 \%)$ in postmenopausal women who did not use estrogen replacement, after adjustment for confounding variables. These effects were strongest in those who used both aspirin and COX2 inhibitors daily. A similar pattern was seen in individuals with osteoporosis, which makes confounding by indication an unlikely explanation for these results.

Men who are current or previous daily users of COX2 inhibitors might benefit from BMD assessment, in order to assess their future fracture risk.

Original article Richards JB et al. (2006) The effect of cyclooxygenase- 2 inhibitors on bone mineral density: results from the Canadian Multicentre Osteoporosis Study. Osteoporos Int 17: 1410-1419

\section{Risk of knee OA correlates with estradiol and 2-hydroxyestrone levels}

Osteoarthritis $(\mathrm{OA})$ is more prevalent among women than men, and typically develops at 40-50 years of age, around the time of menopause. Several lines of evidence implicate sex hormones in arthritis: circulating estradiol levels decline after menopause, but estradiol 